\title{
Intestinal mucosal microbiota composition of patients with acquired immune deficiency syndrome in Guangzhou, China
}

\author{
HAOMING XU ${ }^{1 *}$, ZHITAO OU $^{2 *}$, YONGJIAN ZHOU ${ }^{1}$, YINGFEI LI ${ }^{1}$, HONGLI HUANG $^{1}$, HAILAN ZHAO $^{1}$, \\ JING XU ${ }^{1}$, MEIJUAN LUO ${ }^{3}$, YOULIAN ZHOU ${ }^{1}$ and YUQIANG NIE ${ }^{1}$ \\ ${ }^{1}$ Department of Gastroenterology and Hepatology, Guangzhou Digestive Disease Center, Guangzhou First People's Hospital, \\ School of Medicine, South China University of Technology, Guangzhou, Guangdong 510180; \\ ${ }^{2}$ Department of Internal Medicine, Guangzhou No. 8 People's Hospital, Guangzhou, Guangdong 510180; \\ ${ }^{3}$ Department of Pediatrics, Guangzhou First People's Hospital, School of Medicine, \\ South China University of Technology, Guangzhou, Guangdong 510180, P.R. China
}

Received March 13, 2020; Accepted July 21, 2020

DOI: $10.3892 /$ etm.2021.9822

\begin{abstract}
Acquired immune deficiency syndrome, caused by the human immunodeficiency virus (HIV), has been associated with intestinal dysbiosis, which includes an increase in the number of mucosa-associated pathobionts. In the present study, the intestinal mucosal microbiota patterns of HIV-infected patients were compared with those of healthy individuals in a population from Guangzhou, China. The gut microbiota of intestinal mucosal samples from 12 patients with HIV (transmission routes included sex and intravenous drug abuse) was compared with that of 12 healthy age- and sex-matched controls. Gut microbial communities were profiled via sequencing of the bacterial 16S ribosomal RNA genes. Dysbiosis in HIV-infected individuals was characterized by decreased $\alpha$-diversity, decreased levels of Firmicutes and increased levels of Proteobacteria. Furthermore, low mean counts of Lachnoclostridium, Roseburia, Thauera, Dorea and Roseburia inulinivorans, and high mean counts of Halomonas and Shewanella bacteria, were indicated to be HIV-associated
\end{abstract}

Correspondence to: Professor Yuqiang Nie or Dr Youlian Zhou, Department of Gastroenterology and Hepatology, Guangzhou Digestive Disease Center, Guangzhou First People's Hospital, School of Medicine, South China University of Technology, 1 Panfu Road, Guangzhou, Guangdong 510180, P.R. China

E-mail: eynieyuqiang@scut.edu.cn

E-mail: eyyoulianzhou@scut.edu.cn

*Contributed equally

Abbreviations: AIDS, acquired immune deficiency syndrome; HIV, human immunodeficiency virus; ART, antiretroviral therapy; IDA, intravenous drug abuse; PCA, principal component analysis; LEfSe, linear discriminant analysis effect size; OTU, operational taxonomic units

Key words: acquired immune deficiency syndrome, human immunodeficiency virus, intestinal mucosa, microbiota, disease transmission mucosal bacterial alterations. The relative abundance of Fusobacterium and Lachnoclostridium was significantly decreased, while that of Halomonas and Shewanella was significantly increased in patients with sexually transmitted $\mathrm{HIV}$-infection compared with healthy controls. Alterations of the gut microbiota during HIV infection were also indicated to be associated with the route of HIV transmission. Certain bacteria may be potential biomarkers for HIV infection in patients from Guangzhou, China.

\section{Introduction}

Acquired immunodeficiency syndrome (AIDS), caused by human immunodeficiency virus (HIV), suppresses the human immune system mainly through its effects on the CD4 ${ }^{+} \mathrm{T}$-cell compartment and has been extensively studied since the early 1980s (1). The development of antiretroviral therapy (ART) and highly active ART have markedly reduced HIV-associated morbidity and mortality, but complete eradication of this chronic infection is currently not feasible. Increasing evidence has suggested that the gut microbiota has an important role in the pathogenesis of AIDS, host deterioration and viral transmission (2-4). Studies have indicated increases in commensal bacteria, such as Pseudomonas, in the feces of patients infected with HIV and decreases in Lactobacilli and Bifidobacteria during the early stages of HIV infection, elevating the risk of gut dysbiosis (5). Furthermore, Brenchley et al (6) indicated that microbial translocation is a cause of systemic immune activation in chronic HIV infection and that the levels of certain bacterial products were elevated in HIV-infected individuals.

The gut microbiota has attracted much attention due to its newly discovered role in the maintenance of human health and disease pathogenesis, particularly in association with the gastrointestinal tract (7). Studies have identified alterations in microbial diversity and bacterial composition between the fecal microbiota and intestinal mucosal microbiota of healthy individuals $(3,8)$. Deep-sequencing analysis of healthy volunteers has made it possible to characterize differences between fecal microbiota and intestinal mucosal microbiota (9-11). 
These research studies have also demonstrated that the fecal microbiota and intestinal mucosal microbiota are two distinct microbial communities and that there is a requirement to further investigate them to gain a better understanding of the significance of alterations in the gut microbiota in different diseases. However, most prior studies on the gut microbiota of patients with HIV infection have focused on alterations in the fecal microbiota, while the intestinal mucosal microbiota has been investigated mostly in African and Caucasian populations.

In China, a national HIV epidemic has been declared in 12 of the 31 provinces, and >10,000 individuals were living with HIV or AIDS in 2014 (12). However, studies on the association between the gut microbiota and HIV infection in China are currently limited. In a study performed in Zhejiang province of eastern China, Ling et al (13) indicated that the $\alpha$-diversity indices were not significantly different between healthy controls and patients infected with HIV, while the proportion of Firmicutes/Bacteroidetes was significantly elevated in HIV patients. Sun et al (14) performed a study in Shanghai and determined that the microbiota of individuals infected with HIV had a lower $\alpha$-diversity, with enrichment of Firmicutes and Proteobacteria at the phylum level and suppression of the bacterial class Clostridia and the bacterial families Ruminococcaceae and Lachnospiraceae. A previous study by our group (15), in which stool samples obtained from a population in Guangzhou, China, were analyzed, also indicated that gut dysbiosis was more common among patients with AIDS and was characterized by a lower $\alpha$-diversity, low mean counts of Bacteroidetes, Faecalibacterium, Prevotella, Bacteroides vulgatus, Dialister and Roseburia inulnivorans, as well as elevated mean counts of Proteobacteria, Enterococcus, Streptococcus, Lactobacillus, Lachnoclostridium, Ruminococcus gnavus and Streptococcus vestibularis. However, these studies only analyzed fecal samples and did not explore the intestinal mucosa.

In the present study, for further exploration, the intestinal mucosal microbiota of healthy and HIV-infected individuals from Guangzhou, China was examined. The intestinal mucosa of HIV-infected patients was profiled by sequencing analysis to obtain the composition of the microbiota and identify potential universal and specific biomarkers for HIV infection. Furthermore, differences in the characteristics of the microbiota between patients who had been infected via different routes, i.e. sexual transmission and intravenous drug abuse (IDA), were determined. This investigation has the potential to improve the pathological outcomes and characterize the intestinal mucosal microbiota of HIV-infected individuals.

\section{Materials and methods}

Subjects. In total, $12 \mathrm{HIV}$-infected patients and 12 healthy individuals were enrolled in the present study between March and October 2015 at the Institute for Infectious Diseases, Guangzhou No. 8 People's Hospital, Guangzhou Medical University (Guangzhou, China), for cross-sectional comparison of their intestinal mucosal microbiotas. The diagnosis of the HIV-infected individuals was verified at the Guangzhou Center for Disease Control and Prevention using PCR and
HIV-1 antibody tests. According to the different routes of HIV infection, patients were divided into IDA and sexual transmission, and those who had a history/involvement in both should be excluded. Nine patients were treatment-naïve and 3 patients with highly active antiretroviral therapy. Age-matched and sex-matched healthy volunteers were recruited from the same community (Table I). The exclusion criteria used were an age of $<18$ years, use of antibiotics or probiotics in the previous 4 weeks, a history of inflammatory bowel disease, evidence of hepatitis $\mathrm{B}$ or $\mathrm{C}$ virus infection or other chronic diseases, as well as pregnant or breastfeeding individuals (16). This study was approved by the Ethics Committee of Guangzhou No. 8 People's Hospital, Guangzhou Medical University (Guangzhou, China). All individuals enrolled provided written informed consent to participate.

Sample collection and extraction of bacterial genomic DNA. Terminal ileum mucosal biopsies were obtained during colonoscopy using disposable flexible biopsy forceps. The samples to be used for bacterial genomic DNA extraction were immediately transferred to the laboratory and were stored at $-80^{\circ} \mathrm{C}$. Total DNA was extracted from samples using a DNeasy Blood and Tissue Kit (Qiagen $\mathrm{GmbH}$ ). DNA concentrations were determined using a NanoDrop 2000 Bioanalyzer at $260 \mathrm{~nm}$ (Thermo Fisher Scientific, Inc.). All DNA samples were stored at $-20^{\circ} \mathrm{C}$ prior to PCR amplification and Illumina sequencing was performed.

PCR amplification and Illumina sequencing. The primers, V4-515 forward, 5'-GTGCCAGCMGCCGCGGTAA-3' and V4-806 reverse, 5'-GGACTACHVGGGTWTCTAAT-3', were used to amplify the bacterial $16 \mathrm{~S}$ ribosomal RNA V4 fragments. PCR was performed using Phusion ${ }^{\circledR}$ High-Fidelity PCR Master Mix (New England Biolabs). The PCR products were combined with equal volumes of a loading buffer (containing SYBR-Green) before electrophoresis was performed on $2 \%$ agarose gel. DNA samples between 400 and 450 bp in length were identified as bright strips and were selected for use in subsequent experiments. Sequencing libraries were generated using the TruSeq DNA PCR-Free Sample Preparation Kit (Illumina, Inc.) following the manufacturer's protocol and index codes were added. Library quality was assessed using a Qubit 2.0 Fluorometer (Thermo Fisher Scientific, Inc.) and an Agilent Bioanalyzer 2100 system (Agilent Technologies). Thereafter, the library was sequenced using the Illumina HiSeq 2500 platform (Illumina, Inc.).

Data analysis. The sequences were analyzed using the QIIME software (version 1.7.0) package (17). In-house Perl scripts were used to analyze $\alpha$-diversity within the samples and $\alpha$-diversity among the samples (18). First, reads were filtered out using QIIME quality filters. Subsequently, the program pick_de_novo_otus.py was used to select operational taxonomic units (OTUs) by generating an OTU table. Sequences with $\geq 97 \%$ similarity were assigned to the same OTU. A representative sequence of each OTU was explored for further annotation. An RDP classifier (19) was used to annotate taxonomic information of each representative sequence. $\alpha$-diversity was determined through Shannon, Chao 1, Observed_species and PD_whole_tree indices to analyze the 
A

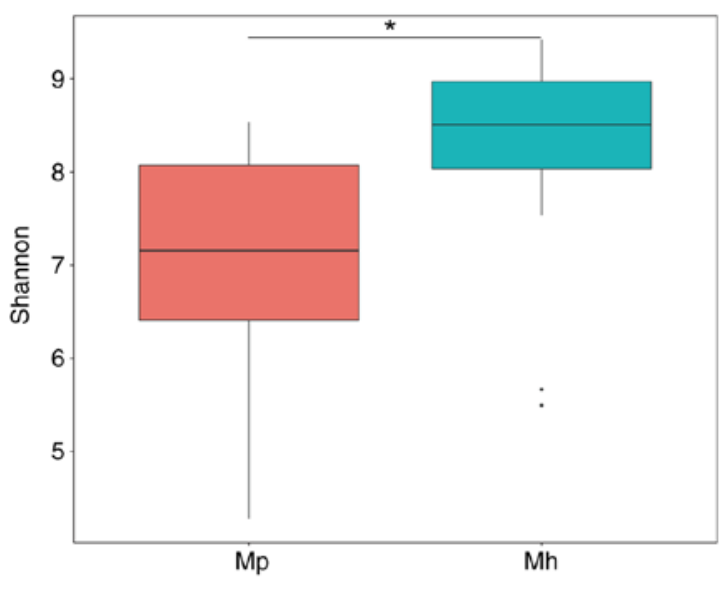

B PCA plot

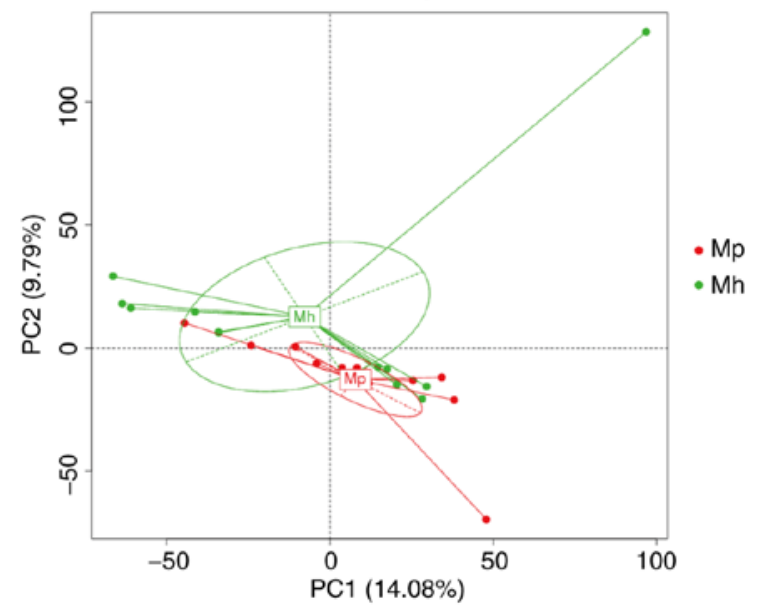

Figure 1. (A) Shannon index demonstrating decreased $\alpha$-diversity in HIV-infected individuals. (B) PCA indicated differences in the clustering of bacterial communities based on HIV infection status. Each dot represents a single mucosal sample. ${ }^{*} \mathrm{P}<0.05$. Mp, HIV patients; Mh, healthy controls. PCA, principal component analysis; HIV, human immunodeficiency virus.

Table I. Baseline clinical characteristics for each group of subjects.

\begin{tabular}{lcc}
\hline Item & $\begin{array}{c}\text { HIV-infected } \\
(\mathrm{n}=12)\end{array}$ & $\begin{array}{c}\text { Healthy controls } \\
(\mathrm{n}=12)\end{array}$ \\
\hline Sex (female/male) & $6 / 6$ & $6 / 6$ \\
Age (years) & $40.0 \pm 10.58$ & $36.1 \pm 9.67$ \\
CD4 ${ }^{+}$cell count $(1 / \mu 1)$ & $94.92 \pm 41.439$ & $\mathrm{NA}$ \\
Viral load (HIV-1 RNA & $5.65 \pm 2.384 \times 10^{5}$ & $\mathrm{NA}$ \\
copies/ml) & & \\
Route of transmission & & \\
Intravenous drug abuse & 3 & $\mathrm{NA}$ \\
Sex & 9 & $\mathrm{NA}$ \\
\hline
\end{tabular}

Values are expressed as the mean \pm standard deviation. ND, not assessed; HIV, human immunodeficiency virus.

complexity of species diversity in each sample (computed with core_diversity_analysis.py script within QIIME) $(17,20)$. Wilcoxon rank-sum test was used to compare the $\alpha$-diversity differences between groups within SPSS (version 22.0; IBM Corp.). Subsequently, $\beta$-diversity was generated within QIIME by using weighted and unweighted Unifrac distance matrices. UniFrac distances are appraised as the distance between bacterial communities explaining phylogenetic relationships between bacteria $(21,22)$. Cluster analysis was performed prior to principal component analysis (PCA), which was applied to reduce the dimension of the original variables, using the FactoMineR package and ggplot2 package in R software (version 2.15.3). Significant differences between specific taxa were analyzed using analysis of variance followed by post-hoc t-tests, with Bonferroni and Benjamini-Hochberg false discovery rate corrections applied for multiple testing. Linear discriminant analysis (LDA) effect size (LEfSe) analysis [http://huttenhower.sph.harvard.edu/lefse/] was used to identify differences in taxa present between groups. First step, the non-parametric factorial Kruskal-Wallis rank-sum test was used to detect taxa with significant differential abundance. Subsequently, biological consistency was investigated using a set of pairwise tests among subclasses using the Wilcoxon rank-sum test. Finally, the Linear Discriminant Analysis (LDA) was used to estimate the effect size of each differentially abundant trait. $\alpha$-values of 0.05 were used for the Kruskal-Wallis sum-rank test and a threshold of 3.6 was chosen for logarithmic LDA scores (23).

\section{Results}

Intestinal mucosal bacterial diversity among HIV patients. Notably, $\alpha$-diversity refers to bacterial diversity, particularly the richness of each taxon within an environment/host, while $\beta$-diversity compares similarities or differences in communities between environments/hosts. Shannon $(\mathrm{P}=0.02418)$, Chao $1(\mathrm{P}=0.03872)$, Observed_species $(\mathrm{P}=0.03324)$ and PD_whole_tree $(\mathrm{P}=0.51370)$ indices were used to compare richness estimators and it was indicated that the Shannon, Chao 1 and Observed_species indices of the intestinal mucosal microbiota were significantly lower compared with those of the healthy controls (Figs. 1A and S1). To reveal the effect of HIV infection on the composition of the microbiota, $\beta$-diversity comparison (PCA) was used to identify similarities in microbial community structure. The results of the PCA suggested that the microbiota of patients with HIV infection differed substantially from that of healthy individuals (Fig. 1B).

Intestinal mucosal bacterial composition in patients with $H I V$. Bacterial community structure was compared between the HIV-infected group and the healthy group. Firmicutes, Proteobacteria, Bacteroidetes and Actinobacteria were the most predominant phyla, which constituted $82 \%$ of the intestinal mucosal samples of HIV-infected patients and healthy controls (Fig. 2A). The proportions of Proteobacteria (42.04 vs. $36.05 \%)$ and Bacteroidetes (17.58 vs. $13.23 \%$ ) were elevated in HIV-infected patients compared with those in the controls, while the proportions of Firmicutes 

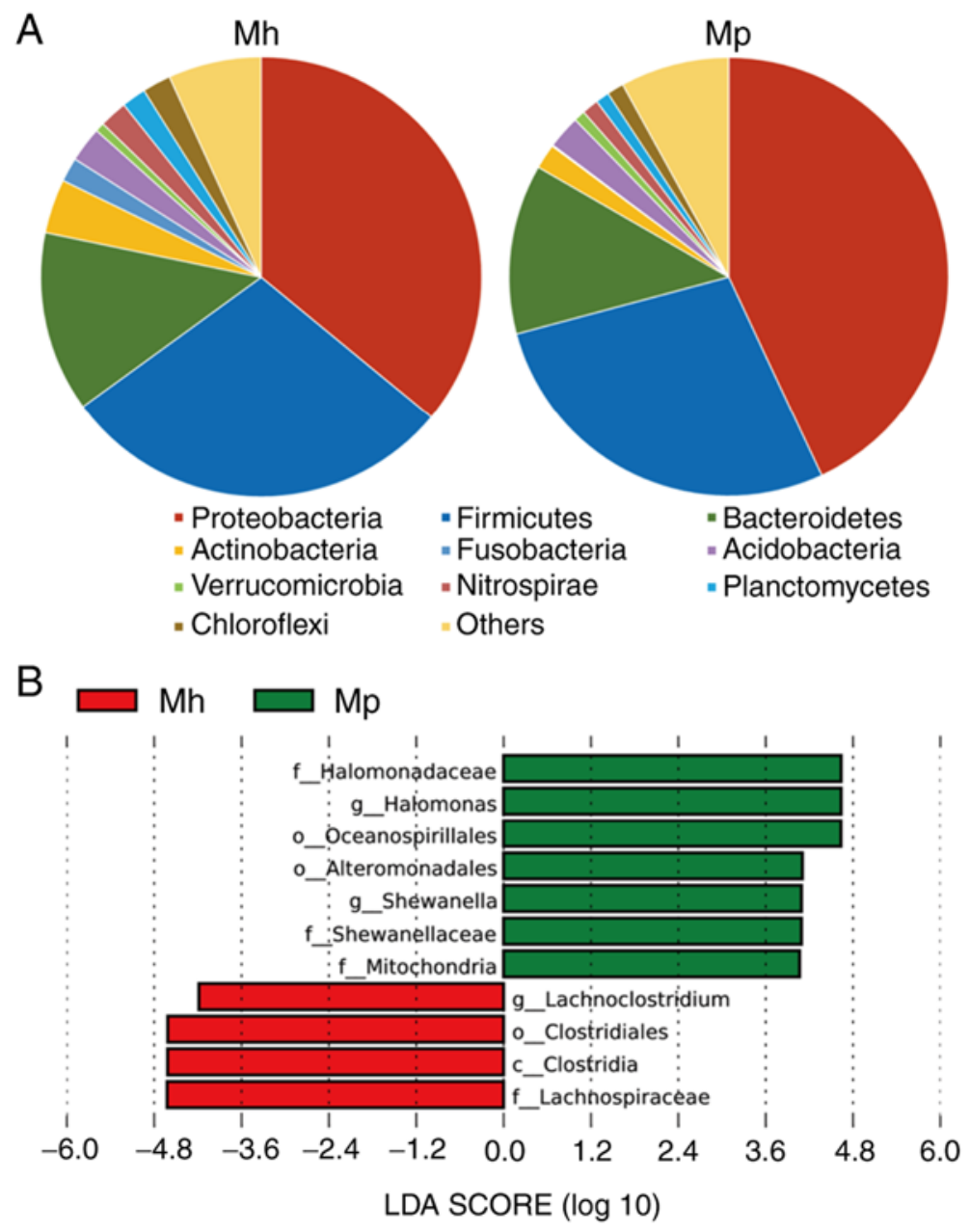

Figure 2. (A) Relative abundance of intestinal mucosal bacteria of different phyla; (B) LDA modeling of the bacterial hierarchy in HIV-infected patients and healthy controls. Groups: Mp, HIV patients; Mh, healthy controls. c_: class; o_: order; f_: family; g_: genus; HIV, human immunodeficiency virus; LDA, linear discriminant analysis.

(22.35 vs. $28.97 \%)$ and Actinobacteria (1.06 vs. $3.99 \%)$ were lower in HIV patients compared with the controls.

For further analysis of changes at lower taxonomic levels (genus level), the microbiota was compared between healthy controls and HIV-infected patients using an LEfSe analysis. This analysis revealed 11 discriminative features (ILDA scorel >3.6). Lachnoclostridium, a Firmicute, was abundant in the healthy control samples, while Halomonas and Shewanella, which are Proteobacteria, were abundant in the samples of HIV-infected patients. At the genus and species levels, Halomonas $(\mathrm{P}=0.008)$ and Shewanella $(\mathrm{P}=0.009)$ were the most abundant in the intestinal mucosal microbiota of HIV patients compared with in the healthy controls, while the levels of Lachnoclostridium ( $\mathrm{P}=0.030)$, Roseburia $(\mathrm{P}=0.047)$, Thauera $(\mathrm{P}=0.033)$, Dorea $(\mathrm{P}=0.015)$ and Roseburia inulinivorans $(\mathrm{P}=0.023)$ were significantly lower in $\mathrm{HIV}$-infected patients than in the healthy controls (Figs. 2B and 3).

Association between the route of HIV transmission and intestinal mucosal microbiota. HIV-infected patients were divided into two groups based on the route of HIV transmission: Sex or IDA. Subsequently, the microbial community structures were compared between these two groups and with the healthy control group. The results indicated that the microbiota differed significantly between healthy group and sexually transmitted HIV-infected group but there were no significant differences between IDA HIV-infected group versus the healthy group or sexually transmitted HIV-infected group (Fig. 4A). Furthermore, LEfSe analysis revealed 17 discriminative features (ILDA scorel $>3.6$ ) in the gut microbiota of individuals with sexually transmitted HIV infection compared with healthy controls and the relative abundances of Fusobacterium and Lachnoclostridium were significantly lower, while those of Halomonas and Shewanella were significantly higher (Fig. 4B).

\section{Discussion}

The present study was the first to compare between the intestinal mucosal microbiota of HIV-infected patients and healthy individuals in a population from Guangzhou, China, using 16s-sequencing. The results demonstrated that the mucosal bacterial communities of HIV-infected patients were suppressed, compared with those of healthy controls. The gut microbiota of HIV-infected patients contained higher proportions of potentially pathogenic bacteria, including Halomonas and Shewanella. In addition, the gut microbiota of HIV-infected patients was composed of lower proportions 
A

$M p \square M h$
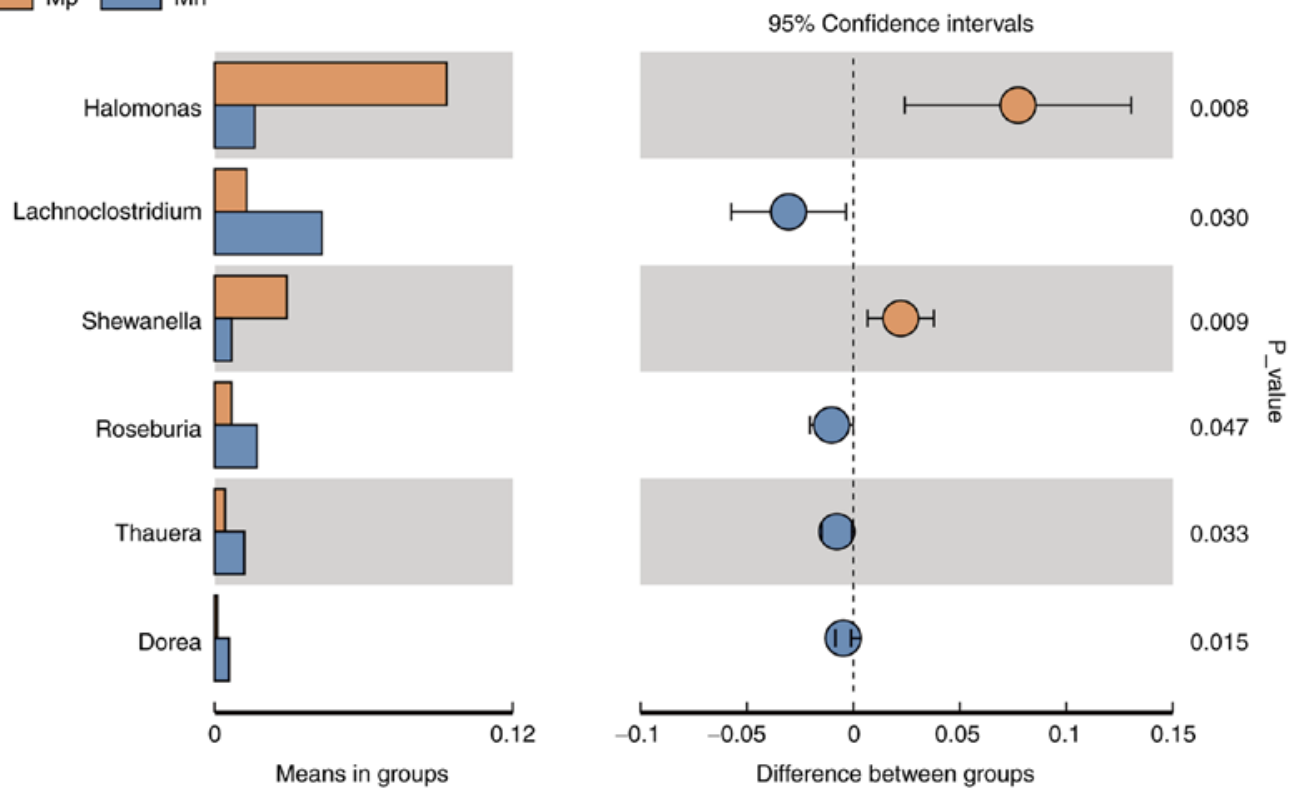

B

$\mathrm{Mp} \square \mathrm{Mh}$
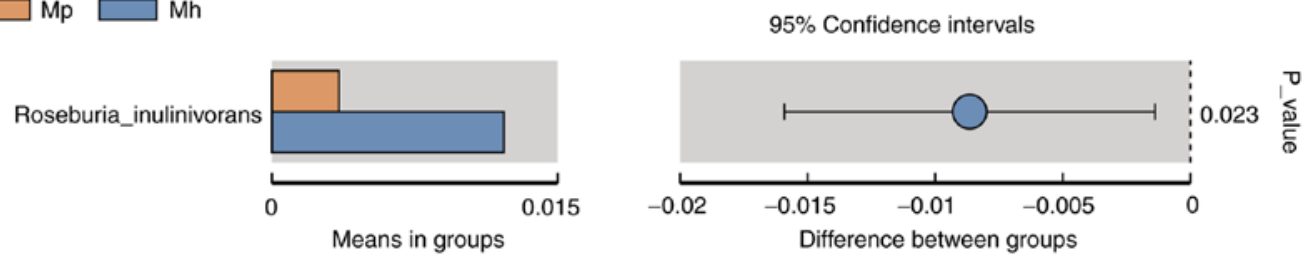

Figure 3. Intestinal mucosal bacterial abundance by (A) genus and (B) species. The mean values of the relative abundances of bacteria in the groups are presented in the left-hand panel, while the differences in relative abundance of bacteria between groups and their P-value are presented in the right-hand panel. Groups: Mp, patients with human immunodeficiency virus; Mh, healthy controls.

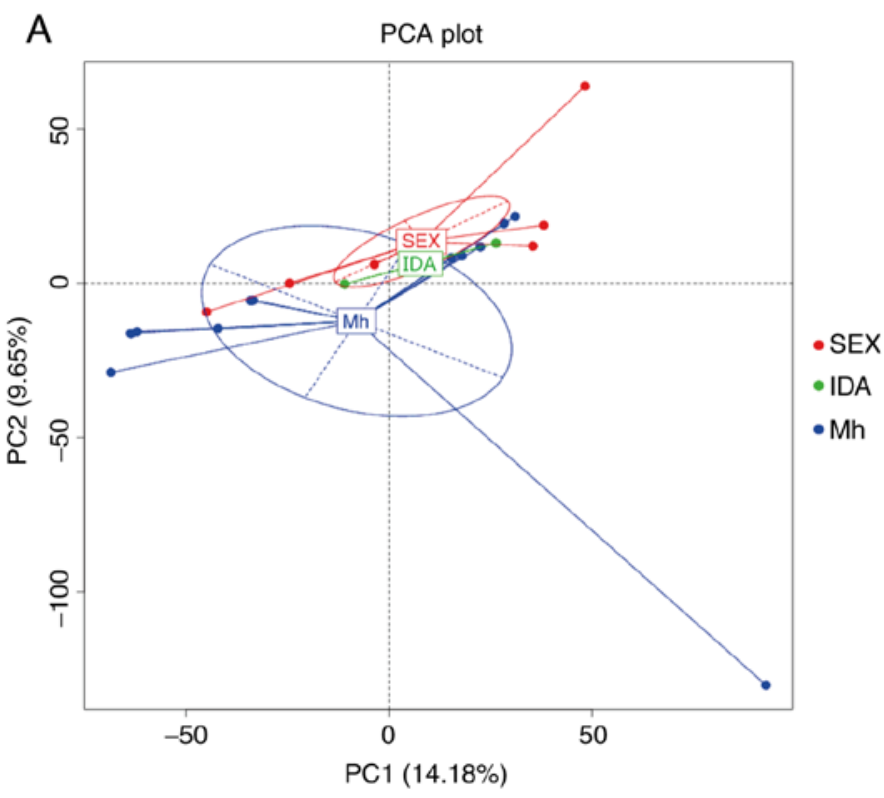

B

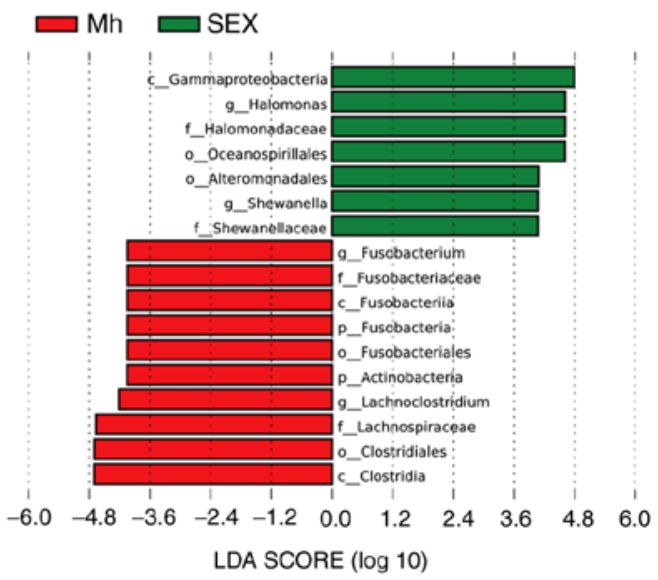

Figure 4. (A) PCA showing differences in the clustering of bacterial communities. (B) LDA modeling of the bacterial hierarchy between sexually transmitted HIV patients and healthy controls. Groups: Sex, patients with sexually transmitted HIV; IDA, HIV infection via intravenous drug abuse; Mh, healthy controls. p_: phylum; c_: class; o_: order; f_: family; g_: genus; HIV, human immunodeficiency virus; LDA, linear discriminant analysis; PCA, principal component analysis.

of Lachnoclostridium, Roseburia, Thauera, Dorea and Roseburia inulinivorans, compared with that of healthy controls.
Compared to a traditional fecal microbiota analysis, changes in the mucosal microbiota are able to more accurately reflect changes in the gut microbiota of a patient's intestine $(24,25)$. 
The samples used in the present study were collected from the end of the ileum and due to anatomical particularities, different mucosal bacteria that were previously underreported were identified. Traditional fecal sample sequencing techniques are likely to be affected by dietary structure and air exposure. Intestinal mucosa, as a part of human tissue, also reflects ethnic differences, which may better reflect colonization of gut microbiota in different populations and cultures. Dinh et al (26) and Lozupone et al (16) assessed American subjects to determine the association between HIV infection and fecal microbial changes. They indicated that the microbiota of HIV-infected individuals in the US was similar to that of healthy individuals from agrarian populations of Malawi and Venezuela. Overall, this noticeable phenomenon indicated that the bacterial composition is also dependent on the environment and diet.

The present study was the first to assess intestinal mucosal samples in patients with HIV from Guangzhou (China). An increased abundance of bacteria of the phylum Proteobacteria, as well as decreased abundance of the phylum Firmicutes, was detected in HIV-infected patients. Dillon et al (3) observed similar changes at the phylum level in mucosal samples of HIV-infected patients. However, the present study focused exclusively on Chinese subjects. The relative abundances of the Proteobacteria, Halomonas and Shewanella were indicated to be higher in the mucosal microbiota of HIV-infected individuals than in healthy subjects. Proteobacteria are known as conditional pathogens that mediate the inflammatory response and may elicit an immune response. Of note, the relative abundances of Halomonas and Shewanella, which are extremely rare in the human gut, were significantly elevated in patients infected with HIV. At present, there are no other reports of these two bacterial strains in the gut microbiota in HIV infected patients, to the best of our knowledge. These two genera of bacteria tend to thrive in seawater and industrial nitrification environments (27-29). Therefore, Halomonas and Shewanella may be specific biomarkers for HIV-infected individuals in Guangzhou, China, particularly in coastal areas.

The present study also provided a comparison of the gut microbiota between HIV-infected patients infected through two different transmission routes to identify pivotal biomarkers that may be associated with the transmission route of HIV infection. In the largest study to date that examined the gut microbiota of HIV-infected individuals, Noguera-Julian et al (30) indicated that a high Prevotella/low Bacteroides enterotype in stool specimens, was highly associated with males who have sex with males, regardless of their HIV infection status. This may explain the perceived association between this enterotype and HIV infection status in prior studies that did not control for sexual behavior. In the present study, the abundances of Fusobacterium and Lachnoclostridium were significantly lower, while those of Halomonas and Shewanella were significantly higher in individuals with sexually transmitted HIV compared with patients with IDA. However, the current results indicated that the microbiota differed significantly between the healthy group and sexually transmitted HIV-infected group but there were no significant differences between the IDA HIV-infected group versus healthy group or sexually transmitted HIV-infected group, the reason is unknown and may be further elucidated in the future. The present study was the first to attempt to compare mucosal microbiota communities between two routes of transmission in a Chinese HIV-infected population.

Several limitations of the present study should be noted. First, the samples were collected from patients who had already been diagnosed with HIV; therefore, the results did not indicate whether the changes in the gut microbiota were a cause or an outcome of HIV infection. HIV infection has been associated with host-microbe interactions, which may trigger the mucosal immune response, and which may have confounded the present results. Since sampling was performed while each patient underwent colonoscopy, the entire sample population was subjected to intestinal cleansing through drinking laxative, which may have led to changes in the gut microbiota. Based on previous studies (31-33), intestinal cleansing may affect the structure of the gut microbiota, leading to changes including decreases in abundances at the family level, causing changes in the abundances of Latobacillaceae and Enterobacteriaceae, as well as at the genus level, causing changes in the abundances of Blautia, Butyricicaccus and Mucispirillum. The results of the present study were not significantly different from those of previous studies. However, the effect of intestinal cleansing on the intestinal microbiota requires to be addressed in future studies. Furthermore, alterations of the gut microbiota are dynamic, and due to a lack follow-up data and a more diverse patient population, it was not possible to evaluate long-term gut microbial changes or include more mucosal samples. A previous study by our group reported that the abundance of Lachnoclostridium was elevated in stool samples of patients with HIV infection (15); however, in the present study, the relative abundance of Lachnoclostridium was lower. The reasons for this difference may be as follows: i) Different sources of samples (feces vs. terminal ileum mucosal biopsies); ii) influence of intestinal preparation (use of laxatives prior to colonoscopy); iii) anti-viral drugs for HIV may have a large influence on the gut microbiota. Villanueva-Millán et al (34) indicated that different anti-viral drugs for HIV produced different effects on the $\alpha$-diversity of HIV patients and these differences were closely associated with changes at lower taxonomic levels (genus level and species level) rather than at the phylum level. Nowak et al (35) reported that anti-AIDS drugs may decrease the levels of the most abundant bacteria, Prevotella, and increase the levels of pathogenic bacteria, including Peptoniphilus, Finegoldia, Anaerococcus and Campylobacter, compared with those in untreated HIV-infected individuals. Therefore, longitudinal and functional studies are required to better understand the role of the gut microbiota of patients with HIV at different treatment stages.

Overall, changes to the gut microbiota during HIV infection are influenced by factors including ethnicity, sex, age, geography, dietary habits, lifestyle, operational factors, sample type and treatment (36-39). In the present study, alterations in the intestinal mucosal microbiota and dysbiosis were identified in HIV-infected patients from Guangzhou, China. The results confirmed that the gut microbiota contains promising biomarkers for non-invasive evaluation of routes of HIV transmission and they may help to develop principles that guide HIV management. Increased abundance of the phylum Proteobacteria and decreased abundance of the phylum 
Firmicutes are important characteristics of the intestinal mucosal microbiota of patients with HIV. The determination of elevated levels of Halomonas and Shewanella may lay a foundation for establishing a set of microbiota-based biomarkers for the diagnosis of HIV. In the future, larger, long-term studies that include metabolomic approaches and culturomics may help identify the association between a specific gut microbiota composition and HIV infection.

In conclusion, the present study indicated that the intestinal mucosal microbiota of patients with HIV had an increased abundance of Proteobacteria and a decreased abundance of Firmicutes bacteria in a population from Guangzhou, China. Certain bacteria, such as Halomonas and Shewanella, may be potential biomarkers for HIV infection in this population. Alterations to the intestinal mucosal microbiota during HIV infection were indicated to be associated with the route of HIV transmission.

\section{Acknowledgements}

Not applicable.

\section{Funding}

This work was supported by grants from the National Natural Science Foundation of China (grant nos. 81700487 and 81871905), the Guangdong Medical Science and Technology Research Fund (grant no. A2019243), the Guangzhou Planned Project of Science and Technology (grant no. 202002030288), the Guangzhou High Technology Project (grant no. 2019GX05), the Fundamental Research Funds for the Central Universities of SCUT (grant no. 2018MS82) and the Guangzhou General Science and Technology Project of Medicine and Health (grant no. 20171A011244).

\section{Availability of data and materials}

The datasets used and/or analyzed during the current study are available from the corresponding author on reasonable request.

\section{Authors' contributions}

$\mathrm{HX}$ and ZO designed the study, collected the clinical data and wrote the manuscript; YJZ interpreted the results; YL and JX collected and carried out the experiments; $\mathrm{HH}, \mathrm{HZ}$ and $\mathrm{ML}$ performed data interpretation and coordinated the revision of the paper. YLZ and YN planned and directed the project and interpreted the results. All authors read and approved the final manuscript.

\section{Ethics approval and consent to participate}

The protocol of the present study was reviewed and approved by the Committee of Guangzhou No. 8 People's Hospital (Guangzhou, China). Written informed consent was obtained from all patients before the research study was conducted.

\section{Patient consent for publication}

No applicable.

\section{Competing interests}

The authors declare that they have no competing interests.

\section{References}

1. Zilberman-Schapira G, Zmora N, Itav S, Bashiardes S, Elinav H and Elinav E: The gut microbiome in human immunodeficiency virus infection. BMC Med 14: 83, 2016.

2. Cohen CR, Lingappa JR, Baeten JM, Ngayo MO, Spiegel CA, Hong T, Donnell D, Celum C, Kapiga S, Delany S, et al: Bacterial vaginosis associated with increased risk of female-to-male HIV-1 transmission: A prospective cohort analysis among African couples. PLoS Med 9: e1001251, 2012.

3. Dillon SM, Lee EJ, Kotter CV, Austin GL, Dong Z, Hecht DK, Gianella S, Siewe B, Smith DM, Landay AL, et al: An altered intestinal mucosal microbiome in HIV-1 infection is associated with mucosal and systemic immune activation and endotoxemia. Mucosal Immunol 7: 983-994, 2014.

4. Doerflinger SY, Throop AL and Herbst-Kralovetz MM: Bacteria in the vaginal microbiome alter the innate immune response and barrier properties of the human vaginal epithelia in a species-specific manner. J Infect Dis 209: 1989-1999, 2014.

5. Gori A, Tincati C, Rizzardini G, Torti C, Quirino T, Haarman M, Ben Amor K, van Schaik J, Vriesema A, Knol J, et al: Early impairment of gut function and gut flora supporting a role for alteration of gastrointestinal mucosa in human immunodeficiency virus pathogenesis. J Clin Microbiol 46: 757-758, 2008.

6. Brenchley JM, Price DA, Schacker TW, Asher TE, Silvestri G, Rao S, Kazzaz Z, Bornstein E, Lambotte O, Altmann D, et al: Microbial translocation is a cause of systemic immune activation in chronic HIV infection. Nat Med 12: 1365-1371, 2006.

7. Blumberg R and Powrie F: Microbiota, disease, and back to health: A metastable journey. Sci Transl Med 4: 137rv7, 2012.

8. ZoetendalEG, von Wright A, Vilpponen-Salmela T,Ben-Amor K, Akkermans AD and de Vos WM: Mucosa-associated bacteria in the human gastrointestinal tract are uniformly distributed along the colon and differ from the community recovered from feces. Appl Environ Microbiol 68: 3401-3407, 2002.

9. Durbán A, Abellán JJ, Jiménez-Hernández N, Salgado P, Ponce M, Ponce J, Garrigues V, Latorre A and Moya A: Structural alterations of faecal and mucosa-associated bacterial communities in irritable bowel syndrome. Environ Microbiol Rep 4: 242-247, 2012.

10. Ringel Y, Maharshak N, Ringel-Kulka T, Wolber EA, Sartor RB and Carroll IM: High throughput sequencing reveals distinct microbial populations within the mucosal and luminal niches in healthy individuals. Gut Microbes 6: 173-181, 2015.

11. Tap J, Derrien M, Törnblom H, Brazeilles R, Cools-Portier S, Doré J, Störsrud S, Le Nevé B, Öhman L and Simrén M: Identification of an intestinal microbiota signature associated with severity of irritable bowel syndrome. Gastroenterology 152: 111-123.e8, 2017.

12. Zhang L, Chow EP, Jing J, Zhuang X, Li X, He M, Sun H, Li X, Gorgens M, Wilson D, et al: HIV prevalence in China: Integration of surveillance data and a systematic review. Lancet Infect Dis 13: 955-963, 2013.

13. Ling $\mathrm{Z}$, Jin $\mathrm{C}, \mathrm{Xie} \mathrm{T}$, Cheng $\mathrm{Y}, \mathrm{Li} \mathrm{L}$ and $\mathrm{Wu} \mathrm{N}$ : Alterations in the fecal microbiota of patients with HIV-1 infection: An Observational Study in A Chinese Population. Sci Rep 6: 30673, 2016.

14. Sun Y, Ma Y, Lin P, Tang YW, Yang L, Shen Y, Zhang R, Liu L, Cheng J, Shao J, et al: Fecal bacterial microbiome diversity in chronic HIV-infected patients in China. Emerg Microbes Infect 5: e31, 2016.

15. Zhou Y, Ou Z, Tang X, Zhou Y, Xu H, Wang X, Li K, He J, Du Y, Wang $\mathrm{H}$, et al: Alterations in the gut microbiota of patients with acquired immune deficiency syndrome. J Cell Mol Med 22: 2263-2271, 2018.

16. Lozupone CA, Li M, Campbell TB, Flores SC, Linderman D, Gebert MJ, Knight R, Fontenot AP and Palmer BE: Alterations in the gut microbiota associated with HIV-1 infection. Cell Host Microbe 14: 329-339, 2013.

17. Caporaso JG, Kuczynski J, Stombaugh J, Bittinger K, Bushman FD, Costello EK, Fierer N, Peña AG, Goodrich JK, Gordon JI, et al: QIIME allows analysis of high-throughput community sequencing data. Nat Methods 7: 335-336, 2010. 
18. Kuczynski J, Stombaugh J, Walters WA, Gonzalez A Caporaso JG and Knight R: Using QIIME to analyze 16S rRNA gene sequences from microbial communities. Curr Protoc Bioinformatics: Chapter 10: Unit 10.7, 2011.

19. Wang Q, Garrity GM, Tiedje JM and Cole JR: Naive Bayesian classifier for rapid assignment of rRNA sequences into the new bacterial taxonomy. Appl Environ Microbiol 73: 5261-5267, 2007.

20. Li B, Zhang X, Guo F, Wu W and Zhang T: Characterization of tetracycline resistant bacterial community in saline activated sludge using batch stress incubation with high-throughput sequencing analysis. Water Res 47: 4207-4216, 2013.

21. Lozupone $\mathrm{C}$ and Knight R: UniFrac: A new phylogenetic method for comparing microbial communities. Appl Environ Microbiol 71: 8228-8235, 2005.

22. Nagpal R, Shively CA, Appt SA, Register TC, Michalson KT, Vitolins MZ and Yadav H: Gut Microbiome Composition in Non-human Primates Consuming a Western or Mediterranean Diet. Front Nutr 5: 28, 2018.

23. Segata N, Izard J, Waldron L, Gevers D, Miropolsky L, Garrett WS and Huttenhower C: Metagenomic biomarker discovery and explanation. Genome Biol 12: R60, 2011.

24. Lo Presti A, Zorzi F, Del Chierico F, Altomare A, Cocca S, Avola A, De Biasio F, Russo A, Cella E, Reddel S, et al: Fecal and mucosal microbiota profiling in irritable bowel syndrome and inflammatory bowel disease. Front Microbiol 10: 1655, 2019

25. Mira-Pascual L, Cabrera-Rubio R, Ocon S, Costales P, Parra A Suarez A, Moris F, Rodrigo L, Mira A and Collado MC: Microbial mucosal colonic shifts associated with the development of colorectal cancer reveal the presence of different bacterial and archaeal biomarkers. J Gastroenterol 50: 167-179, 2015.

26. Dinh DM, Volpe GE, Duffalo C, Bhalchandra S, Tai AK, Kane AV, Wanke CA and Ward HD: Intestinal microbiota, microbial translocation, and systemic inflammation in chronic HIV infection. J Infect Dis 211: 19-27, 2015.

27. Gasperotti AF, Revuelta MV, Studdert CA and Herrera Seitz MK Identification of two different chemosensory pathways in representatives of the genus Halomonas. BMC Genomics 19: 266, 2018.

28. Janda JM and Abbott SL: The genus Shewanella: From the briny depths below to human pathogen. Crit Rev Microbiol 40: 293-312, 2014

29. Long MR, Zhang DF, Yang XY, Zhang XM, Zhang YG, Zhang YM, Zhu H and Li WJ: Halomonas nanhaiensis sp. nov., a halophilic bacterium isolated from a sediment sample from the South China Sea. Antonie van Leeuwenhoek 103: 997-1005, 2013.
30. Noguera-Julian M, Rocafort M, Guillén Y, Rivera J, Casadellà M, Nowak P, Hildebrand F, Zeller G, Parera M, Bellido R, et al: Gut microbiota linked to sexual preference and HIV infection. EBioMedicine 5: 135-146, 2016.

31. Drago L, Toscano M, De Grandi R, Casini V and Pace F. Persisting changes of intestinal microbiota after bowel lavage and colonoscopy. Eur J Gastroenterol Hepatol 28: 532-537, 2016.

32. Vich Vila A, Collij V, Sanna S, Sinha T, Imhann F, Bourgonje AR, Mujagic Z, Jonkers DMAE, Masclee AAM, Fu J, et al: Impact of commonly used drugs on the composition and metabolic function of the gut microbiota. Nat Commun 11: 362, 2020.

33. Tropini C, Moss EL, Merrill BD, Ng KM, Higginbottom SK, Casavant EP, Gonzalez CG, Fremin B, Bouley DM, Elias JE, et al: Transient osmotic perturbation causes long-term alteration to the gut microbiota. Cell 173: 1742-1754.e1717, 2018.

34. Villanueva-Millán MJ, Pérez-Matute P, Recio-Fernández E, Lezana Rosales JM and Oteo JA: Differential effects of antiretrovirals on microbial translocation and gut microbiota composition of HIV-infected patients. J Int AIDS Soc 20: 21526, 2017.

35. Nowak RG, Bentzen SM, Ravel J, Crowell TA, Dauda W, Ma B, Liu H, Blattner WA, Baral SD and Charurat ME; TRUSTRV368 Study Group: Rectal microbiota among HIV-uninfected, untreated HIV, and treated HIV-infected in Nigeria. AIDS 31: 857-862, 2017.

36. Ashuro AA, Lobie TA, Ye DQ, Leng RX, Li BZ, Pan HF and Fan YG: Review on the alteration of gut microbiota: The role of HIV infection and old age. AIDS Res Hum Retroviruses 36: 556-565, 2020.

37. Bayigga L, Kateete DP, Anderson DJ, Sekikubo M and Nakanjako D: Diversity of vaginal microbiota in sub-Saharan Africa and its effects on HIV transmission and prevention. Am J Obstet Gynecol 220: 155-166, 2019.

38. Hager CL and Ghannoum MA: The mycobiome in HIV. Curr Opin HIV AIDS 13: 69-72, 2018.

39. Tuddenham S, Koay WL and Sears C: HIV, sexual orientation, and gut microbiome interactions. Dig Dis Sci 65: 800-817, 2020.

This work is licensed under a Creative Commons Attribution-NonCommercial-NoDerivatives 4.0 International (CC BY-NC-ND 4.0) License. 